\title{
Grupo de Pesquisa INTERA - Inteligência em Tecnologias Educacionais e Recursos Acessíveis
}

\author{
Juliana Cristina Braga ${ }^{1}$, Silvia Cristina Dotta ${ }^{1}$, Edson Pimentel ${ }^{1}$, Beatriz Stransky ${ }^{2}$ \\ Ferreira, Lucia Regina Horta ${ }^{1}$, Itana Stiubiener ${ }^{1}$, Natalia Pirani Ghilardi Lopes ${ }^{1}$, \\ Luciano Silva ${ }^{3}$, Nizam Omar ${ }^{3}$ \\ ${ }^{1}$ Universidade Federal do ABC (UFABC) - Santo André - SP \\ ${ }^{2}$ Universidade Federal do Rio Grande do Norte (UFRN) - Natal - RN \\ ${ }^{3}$ Universidade Presbiteriana Mackenzie - São Paulo - SP \\ \{juliana.braga, silvia.dota, edson.pimentel, lucia.horta, \\ itana.stiubiener, natalia.lopes\}@ufabc.edu.br, bia.stransky@gmail.com, \\ luciano.silva.sp@gmail.com, omar@mackenzie.br \\ O grupo de pesquisa INTERA tem como objetivos principais: a) investigar e \\ desenvolver os fundamentos teórico-metodológicos para o desenvolvimento de TICs \\ (Tecnologias de Informação e Comunicação) aplicadas à Educação, considerando os \\ princípios da acessibilidade, da interação e da dialogia; b) avaliar a influência das TICs \\ na aprendizagem; c) produzir TICs por meio de processos iterativos e interativos, de \\ modo a contribuir com a articulação multidisciplinar das áreas envolvidas com a \\ produção, a pesquisa e o uso das TICs na Educação; d) pesquisar o uso das técnicas e \\ métodos de representação e processamento do conhecimento (e informação) para o \\ compartilhamento seguro das TICs na educação.
}

